\title{
Defensive Vocalizations and Motor Asymmetry Triggered by Disinhibition of the Periaqueductal Gray in Non-human Primates
}

\author{
Patrick A. Forcelli ${ }^{1,2,3 *}$, Hannah F. Waguespack ${ }^{1}$ and Ludise Malkova ${ }^{1,2 *}$ \\ ${ }^{1}$ Department of Pharmacology and Physiology, Georgetown University, Washington, DC, USA, ${ }^{2}$ Interdisciplinary Program in \\ Neuroscience, Georgetown University, Washington, DC, USA, ${ }^{3}$ Department of Neuroscience, Georgetown University, \\ Washington, DC, USA
}

OPEN ACCESS

Edited by:

Hisao Nishijo,

University of Toyama, Japan

Reviewed by:

Norberto Cysne Coimbra,

University of São Paulo, Brazil

Rafael Souto Maior,

University of Brasilia, Brazil

*Correspondence:

Patrick A. Forcelli

paf22@georgetown.edu

Ludise Malkova

malkoval@georgetown.edu

Specialty section: This article was submitted to

Systems Biology,

a section of the journal

Frontiers in Neuroscience

Received: 04 January 2017 Accepted: 13 March 2017

Published: 29 March 2017

Citation:

Forcelli PA, Waguespack HF and

Malkova L (2017) Defensive Vocalizations and Motor Asymmetry

Triggered by Disinhibition of the

Periaqueductal Gray in Non-human

Primates. Front. Neurosci. 11:163.

doi: 10.3389/fnins.2017.00163
Rapid and reflexive responses to threats are present across phylogeny. The neural circuitry mediating reflexive defense reactions has been well-characterized in a variety of species, for example, in rodents and cats, the detection of and species-typical response to threats is mediated by a network of structures including the midbrain tectum (deep and intermediate layers of the superior colliculus [DLSC]), periaqueductal gray (PAG), and forebrain structures such as the amygdala and hypothalamus. However, relatively little is known about the functional architecture of defense circuitry in primates. We have previously reported that pharmacological activation of the DLSC evokes locomotor asymmetry, defense-associated vocalizations, cowering behavior, escape responses, and attack of inanimate objects (Holmes et al., 2012; DesJardin et al., 2013; Forcelli et al., 2016). Here, we sought to determine if pharmacological activation of the PAG would induce a similar profile of responses. We activated the PAG in three awake, behaving macaques by microinfusion of GABA-A receptor antagonist, bicuculline methiodide. Activation of PAG evoked defense-associated vocalizations and postural/locomotor asymmetry, but not motor defense responses (e.g., cowering, escape behavior). These data suggest a partial dissociation between the role of the PAG and the DLSC in the defense network of macaques, but a general conservation of the role of PAG in defense responses across species.

Keywords: defensive behavior, macaque, PTSD, primate, GABA-A, bicuculline

\section{INTRODUCTION}

Rapid and reflexive responses to threats are present across phylogeny. These defensive reactions are manifest in species-typical ways; for example, rodents display freezing both to looming stimuli and to unconditioned fear-evoking stimuli, such as fox odor (Cattarelli and Chanel, 1979; Wallace and Rosen, 2000; Yilmaz and Meister, 2013; Shang et al., 2015; De Franceschi et al., 2016). By contrast, primates display alarm calls, avoidance and escape behaviors toward unconditioned fearprovoking stimuli, such as snakes (Izquierdo and Murray, 2004; Shibasaki et al., 2014; Weiss et al., 2015; Kawai and Koda, 2016). In humans, midbrain regions (encompassing the periaquaductal gray and the deep/intermediate layers of the superior colliculus) parametricaly encoded proximity

Abbreviations: DLSC, deep and intermediate layers of the superior colliculus; PAG, periaqueductal gray; BIC, bicuculline methiodide. 
of threating stimuli (Mobbs et al., 2010) and looming threats (Coker-Appiah et al., 2013). The brain networks subserving these behaviors have been well-characterized in rodents and rely critically on the interaction between mid-brain structures (e.g., the superior and inferior colliculi, the periaqueductal gray) and limbic regions (e.g., the amygdala) (Brandão et al., 1994, 2003; Eichenberger et al., 2002; Schenberg et al., 2005; Coimbra et al., 2006; Ullah et al., 2015). However, less is known about the networks controlling defensive responses in primates.

We have recently reported that selective pharmacological activation of the deep and intermediate layers of the superior colliculus (DLSC) evokes a constellation of defensive behaviors (DesJardin et al., 2013). Microinjection of the GABA-A receptor antagonist, bicuculline methiodide, into the DLSC evoked, in a dose-dependent manner, cowering behaviors, explosive escape reactions, alarm vocalizations, and attack of inanimate objects. These behaviors co-occurred with a concurrent reduction in affiliative social interactions when animals were examined in social dyads. Interestingly, transient pharmacological inhibition of the amygdala was able to attenuate some (i.e., cowering) but no other (escape, vocalizations) behaviors evoked by disinhibition of the DLSC (Forcelli et al., 2016). These reflexive behaviors bear striking similarity to some behaviors reported in rodents after activation of the DLSC; for example, rodents likewise display explosive escape behaviors and postural asymmetry following activation of DLSC (Dean et al., 1986; Sahibzada et al., 1986). However, the occurrence of these striking defensive responses in the primate was somewhat surprising given that despite a long history of microinjection into the primate SC, defensive responses had not been previously reported. The fact that these behaviors were not reported (prior to our study) in primates led to the proposal by Redgrave and Dean that emphasized a preferential role of primate DLSC for approach responses rather than defensive responses (Dean et al., 1989). However, our findings indicate that the function of DLSC as a substrate for responding to threats is conserved across species.

Given our finding that the role of DLSC in defensive responses appears to be conserved between rodents and primates, we next sought to examine the role of a sub-adjacent structure, the periaquaductal gray. In rodents, activation of the PAG triggers freezing responses (Bandler et al., 1985a; Krieger and Graeff, 1985). Moreover, in cats, vocalization, defense, and orienting reactions have been reported after PAG activation (Bandler and Carrive, 1988). In the macaque, while vocalizations have been reported, defensive responses have not been reported after PAG activation (Larson and Kistler, 1986). However, anxious temperament in macaques is correlated with increased brain metabolism in PAG as measured by FDG-PET (Fox et al., 2008). Further supporting a role for this structure across species, neuroimaging studies in humans have revealed a topography of PAG activation in response to viewing an aversive image, with large magnitude signal changes seen in the caudal ventromedial and ventrolateral PAG, and the rostral lateral PAG (Satpute et al., 2013). Perhaps most interestingly, electrical stimulation of a medial zone of the dorsolateral tegmentum (including the periaquauctal gray and deep portions of the corpora quadrigemina) in humans has been reported to induce feelings of fright (Nashold et al., 1969).

Thus, based on the above findings, we sought to determine if activation of the lateral PAG in macaques, by microinjection of the GABA-A receptor antagonist, bicuculline methiodide, would elicit defensive responses. We consider the evoked responses in light of our prior findings in the DLSC (Holmes et al., 2012; DesJardin et al., 2013; Forcelli et al., 2016).

\section{METHODS}

\section{Animals}

Three pigtail macaques (Macaca nemestrina) were used in this study, 1 female (JA) and 2 male (GW, ZK). These animals were raised in the Infant Primate Research Laboratory at the University of Washington Regional Primate Research Facility, in a way similar to that described previously (Novak and Sackett, 1997). At $\sim 6$ months of age, the animals were transferred to Georgetown University where all experimental procedures were conducted. Animals were pair-housed within two joined individual cages (size: $61 \times 74 \times 76 \mathrm{~cm}$ each) in a temperature $\left(24^{\circ} \mathrm{C}\right)$ and humidity controlled room with a standard 12 -h light/dark cycle.

When not performing concurrent cognitive testing, animals were given full feed (Primate Lab Diet, \#5049, Purina Mills Inc. International, Brentwood, MD) supplemented with fresh fruit. Water was also available ad libitum in the home cage. Care and housing of the monkeys met or exceeded the standards as stated in the Guide for Care and Use of Laboratory Animals (National Research Council U.S., Institute for Laboratory Animal Research U.S., and National Academies Press U.S., 2011), ILAR recommendations and AAALAC accreditation standards. The study was conducted under a protocol approved by the Georgetown University Institutional Animal Care and Use Committee.

The present experiments began after the animals were extensively socialized and behaviorally trained (including chairtraining), at approximately 2 years of age. In addition to the experimental procedures described here, all subjects were trained on various cognitive tasks administered at the Wisconsin General Testing Apparatus; the tasks included visual object discrimination, visual delayed non-matching to sample, crossmodal auditory-visual matching task, and reinforcer devaluation. As part of those experiments, some animals received drug infusions in BLA (animals JA, and ZK) (Wellman et al., 2005). Additionally, all of these animals received microinjections in the BLA and/or CeA for two other studies of social behavior (Wellman et al., 2016; Forcelli et al., 2017), and two of the animals also received injections into the DLSC (animals ZK and GW) (DesJardin et al., 2013). For these prior studies, the GABAA receptor agonist muscimol, the GABA-A receptor antagonist bicuculline methiodide, the NMDA receptor antagonist AP-7 or the AMPA receptor antagonist NBQX were injected into the sites described. As documented by the histological evaluation of all the cases, damage to the amygdala (Wellman et al., 2016, Figure 1); (Forcelli et al., 2017, Figure 1) or to DLSC (DesJardin et al., 2013, Figure 1) due to insertion of the cannula was minimal. 


\section{Surgical Implantation of Cranial Infusion Platform and Localization of Infusion Sites}

Monkeys were implanted with stereotaxically positioned chronic infusion platforms as we have described extensively elsewhere an (Wellman et al., 2005, 2016; West et al., 2011; Holmes et al., 2012; DesJardin et al., 2013; Dybdal et al., 2013; Forcelli et al., 2014, 2016; Malkova et al., 2015). This platform enabled us to target the periaqueductal gray based on the coordinates assessed by structural magnetic resonance imaging (MRI). Prior to surgery, each monkey received a T1-weighted MRI scan to enable precise placement of the platform. The infusion platform was implanted under anesthesia and aseptic conditions, with postoperative analgesics and antibiotics determined in consultation with the facility veterinarian.

Postoperatively, each monkey received at least one T1-weighted scan with tungsten microelectrodes (FHC, Bowdoinham, ME) placed dorsal to the infusion sites calculated based on the pre-operative MRI. The position of these electrodes, which were visible on the scan, were then used to adjust the final infusion coordinates as needed. Our platform allows for $2 \mathrm{~mm}$ resolution in the anteroposterior and mediolateral planes, and sub-mm resolution in the dorsoventral plane.

\section{Drug Solutions and Intramesencephalic Infusions}

The $\mathrm{GABA}_{\mathrm{A}}$ antagonist bicuculline methiodide (BMI; SigmaAldrich) was dissolved in saline and injected at a dose of 2.5$7 \mathrm{nmol}$ in $0.5-1 \mu \mathrm{l}$ volume, unilaterally. Drug infusions were performed aseptically, while the monkey was seated in a standard primate chair (Crist Instruments, Inc.) with minimal restraint. Infusions were performed using procedures we have previously described (Malkova et al., 2015). A sterile injector cannula was acutely placed into the PAG using the pre-determined coordinates. This 27-gauge cannula was connected, via sterile tubing, to a Hamilton syringe controlled by an infusion pump. The pump was calibrated to deliver solution at a rate of $1 \mu \mathrm{l} / 5$ min. After completion of infusion, the cannula was left in place for 1-5 min prior to removal, to minimize drug reflux up the cannula track. The entire infusion procedure lasted 10-15 min. Behavioral observation was initiated within 15 min following an infusion.

\section{Behavioral Assessment}

Twenty-four hour prior to each drug infusion, the experimental subject was placed into an observation cage $(61 \times 74 \times 76 \mathrm{~cm})$ and video-taped for $30 \mathrm{~min}$; these "baseline" sessions serve as our control. For observation after drug infusion, animals were again transferred to an observation cage and video-taped for 60 min immediately following the completion of drug infusion. The observation cage was placed in room separate from the normal primate housing room and contained no other animals. Consistent with our prior analysis of defensive behaviors evoked from the DLSC (DesJardin et al., 2013), we identified the peak bin (either $0-15 \mathrm{~min}$ or $15-30 \mathrm{~min}$ ) for each infusion and used this single bin for statistical analysis. This allows for slight differences in positioning of infusions across subjects. We did not include later bins, because of potential drug diffusion outside of the structure of interest.

Videotapes were analyzed using the software program The Observer (Noldus Information Technology, Wageningen, Netherlands) according to an ethogram consisting of the behavioral categories we have previously described (Holmes et al., 2012; DesJardin et al., 2013; Forcelli et al., 2016). In addition, we recorded the presence of postural/locomotor asymmetries (quadrupedal circling, head deviation greater than 45 degrees from the midline). A list of operational definitions for the behavioral categories is provided in Table 1. Scores of one observer were used for statistical analysis, however, additional observers were trained to achieve a high level of inter-observer correlation ( $r=0.9$ or better) and analyzed a subset of videotapes.

\section{Histology}

Animals were perfused and brains processed for localization of infusion sites, as we have previously described (Wellman et al., 2005; Dybdal et al., 2013; Forcelli et al., 2014). Representative photomicrographs for each subject are shown in Figure 1.

\section{Analysis of Vocalizations}

We performed a power spectral analysis on three call types from one subject (JA). Calls were isolated from the video/audiorecords and transferred to a PC. Sections containing vocalizations in the absence of other room noise were exported as WAV files and imported into LabChart Pro (Version 8.12, AD Instruments). The data were subjected to a fast Fourier transform (1024 bin frequency resolution; Hann window, 93.75\% window overlap), and the resulting power spectral density was plotted using LabChart functions.

\section{Statistical Analysis}

Data were analyzed using GraphPad Prism (GraphPad Software, Inc, La Jolla, CA). Because defensive behaviors rarely (vocalization, escape; 1 of 6 cases) or never (cowering, attack) occurred under baseline conditions, data were not normally distributed. Thus, a one-tailed Wilcoxon's signed rank test for matched pairs to test the a priori hypothesis that drug treatment $>$ baseline. $P$-values $<0.05$ were considered to be statistically significant.

\section{RESULTS}

We injected three sites in JA, two sites in GW and one site in ZK. Injection sites and drug doses for each site are shown in Table 2. These infusions were all placed in the lateral/dorsolateral PAG. Representative photomicrographs showing cannula tracks are shown in Figure $\mathbf{1}$ for the three animals that were available for histology (GW, JA and ZK). Infusions were targeted at the rostrocaudal level of the intra-aural line and the intended infusion zone is also shown in Figure 1. The latency to the onset of behavioral responses was 15 min or less in all subjects; indeed in three of the six cases behavioral responses to bicuculline microinjection were observed immediately at the start of the observation period (JA-58, JA-66, and ZK-70). 
TABLE 1 | Operational definitions of observed behaviors.

\begin{tabular}{|c|c|}
\hline Behavior & Description \\
\hline Defense-associated vocalizations & Calls consisting of barks and screams[this excludes affiliative vocalizations, such as coos] \\
\hline Cower & Withdrawal to the periphery of the cage in a crouched or recoiled position with an upward directed gaze \\
\hline Escape & Sudden movement/startle response, typically consisting of moving explosively from one side of the cage to the other \\
\hline Attack of inanimate objects & Bitting, hitting, or throwing objects such as toys and/or ratting cage bars \\
\hline Motor/postural asymmetry & Quadrupedal circling, head deviation greater then a45 degree from the midline \\
\hline
\end{tabular}

Behaviors that were coded from video-records.

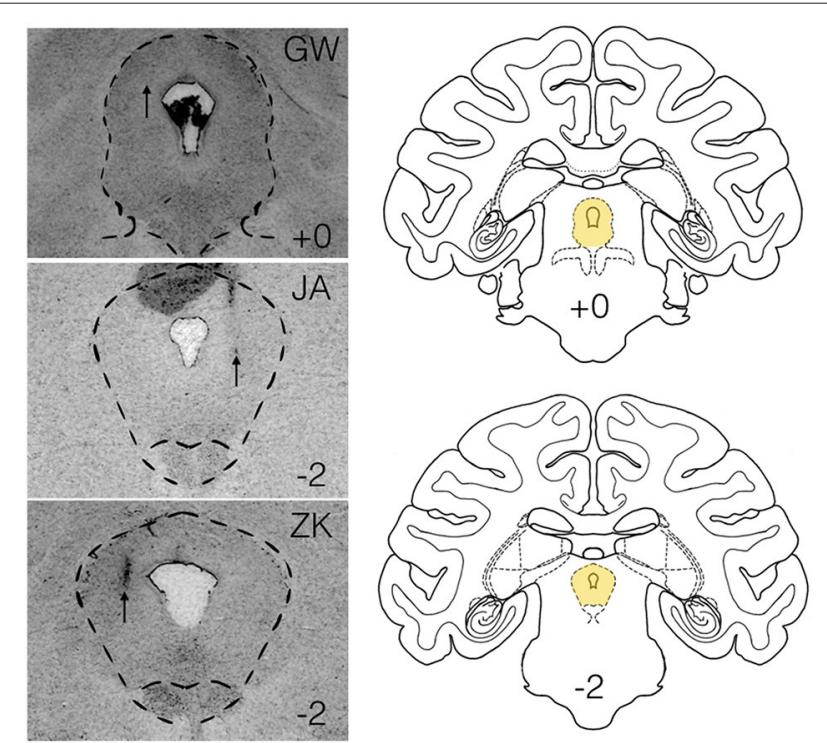

FIGURE 1 | Histological verification of infusion sites within the lateral PAG. Panels on the left show infusion sites in the periaqueductal gray of the three subjects (GW, JA, ZK). Outlined region shows the PAG. Atlas panels on the right show the level of infusion sites for GW and JA (top, +0) and ZK (bottom, -2) relative to the intra-aural line. Atlas panels are from the National Institute of Mental Health/Laboratory of Neuropsychology standard Rhesus Macaque atlas.

Under baseline conditions, defense-associated vocalizations were observed in only one session (JA-58) and occurred at a low rate. Figure 2 shows representative spectrograms for defenseassociated vocalizations (scream, bark). After microinjection of bicuculline into the PAG, vocalizations emerged in all 6 cases. These vocalizations were characterized by barks and screams, with a mean of 148 vocalizations during the 15 -min observation session. These data are shown in Figure 3A. In the one case where vocalizations were observed in the baseline session, the rate of vocalization increased 2.5-fold after bicuculline injection. Coo vocalizations (Figure 2) never occurred after activation of PAG; in one case, they were observed under baseline conditions (JA), but abolished after bicuculline infusion into the PAG. Wilcoxon test revealed a significant increase in vocalizations after bicuculline injection in PAG $(W=21, P=0.0156)$.

During the same session, we quantified cowering behavior, escape behaviors, and attack of inanimate objects. Cowering behavior (Figure 3B) was never present under baseline
TABLE 2 | Infusion sites and drug doses by case.

\begin{tabular}{lcc}
\hline Case & Site (depth) & Dose (nmol) \\
\hline GW-30 & $1(39.5)$ & 5 \\
GW-4 & $1(38.5)$ & 7 \\
ZK-70 & $2(41)$ & 2.5 \\
JA-66 & $3(42.5)$ & 2.5 \\
JA-59 & $3(42.5)$ & 2.5 \\
JA-58 & $3(42)$ & 2.5 \\
\hline
\end{tabular}

Case indicate the experimental subject (initials GW, ZK, JA) and numbers indicate the drug infusion within each animal (e.g., JA-58 was the 58th injection in subject JA). Site indicates a particular antero-posterior and medio-laterally defined location within each subject (See Figure 1); depth indicates the penetration depth within a particular track. Dose indicates the amount of bicuculline methiodide microinfused in each case.

conditions. After bicuculline microinjection into the PAG, cowering emerged in three of the six cases, however, it was rare and accounted for less than $2 \%$ of the observation session even in the most frequent case. Thus, the occurrence of cowering did not differ significantly between baseline and bicuculline-infused sessions $(W=6, P=0.25)$.

Escape behavior (Figure 3C) was present in one of six baseline sessions (JA-58), and two of six bicuculline-infused sessions (JA58 and GW-4). Again, the occurrence of this behavior was rare, even when observed. In the case of GW-4, it is worth noting that this was the highest dose of bicuculline delivered in the present study $(7 \mathrm{nmol})$, yet this led to only 4 escape responses during the 15 min observation segment. The occurrence of escape behaviors did not differ between baseline and drug-infused sessions $(W=$ 3, $P=0.5$ ).

As with cowering behavior, attack of inanimate objects (Figure 3D) never occurred under baseline conditions. After bicuculline infusion into the PAG, attack behaviors emerged in three cases (JA-66, JA-58, and GW-4). The occurrence of this behavior, even when observed, was rare in the female subject (1x in JA-66 and JA-58) and more frequent, but still rare in the male subject (GW-4; 17 counts). Again, it is worth noting that case GW-4 received the highest dose of bicuculline in the present study. The occurrence of attack behaviors did not differ significantly between baseline and drug infused sessions $(W=6$, $P=0.25$ ).

As shown in Figure 4, bicuculline microinjection into the PAG resulted in the emergence of striking motor/postural asymmetries. Data are presented as the duration of asymmetry during the injected session minus the duration of asymmetry 

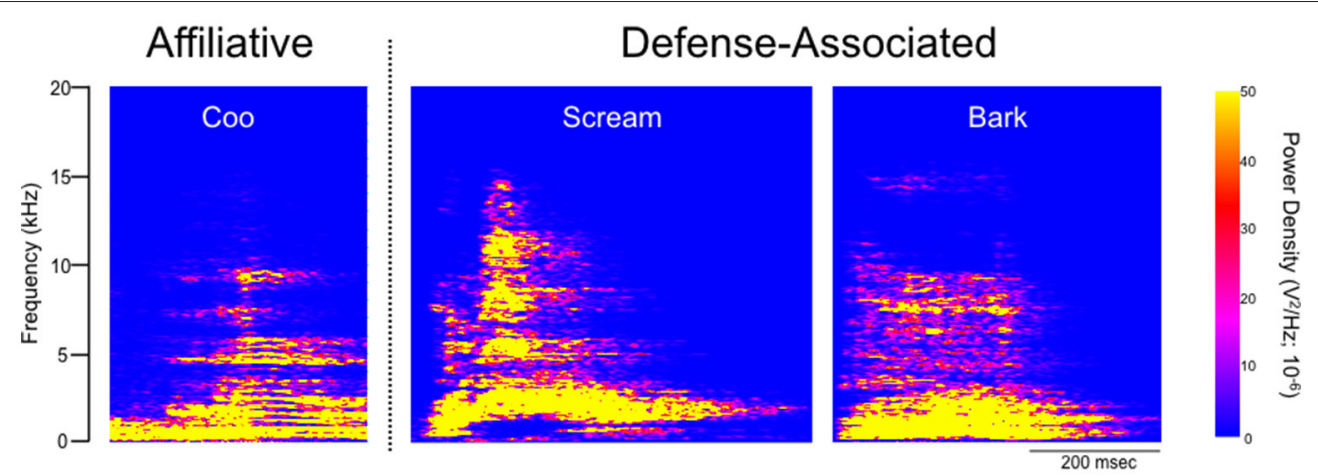

FIGURE 2 | Spectrographic analysis of macaque calls. Panels show representative spectrograms for affiliative calls (coo) and defense-associated vocalizations (scream, bark) in a single subject (JA). JA received $2.5 \mathrm{nmol}$ of bicuculline methiodide in PAG.
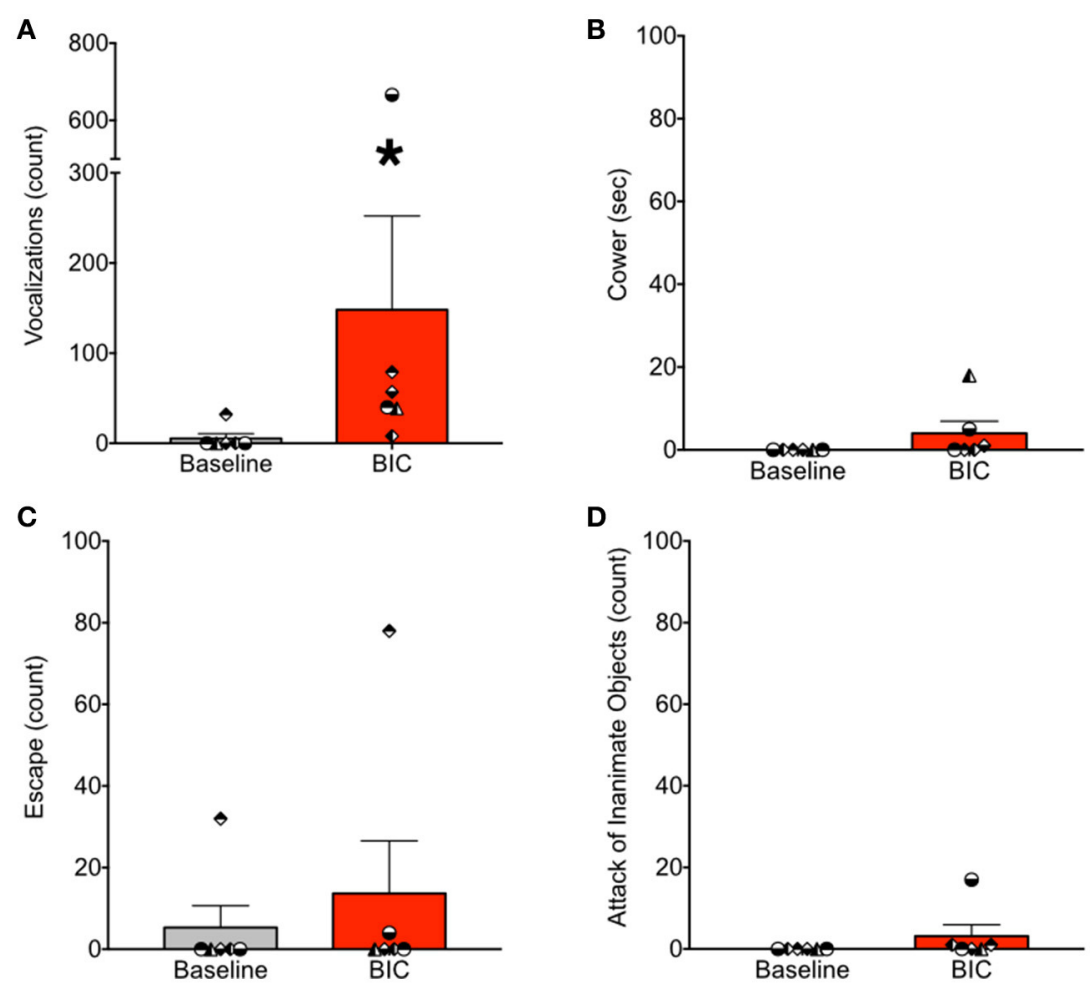

eGW-30

OGW-4

$\Delta Z K-70$

$\triangle \mathrm{JA}-66$

$\triangle \mathrm{JA}-59$

$\diamond \mathrm{JA}-58$

FIGURE 3 | Bicuculline microinjection in PAG increases defense-associated vocalizations but not motor defense responses. (A) Number of defense-associated vocalizations over the course of the 15 min observation segment. (B) Duration of cowering (seconds) over the course of the 15 min observation segment. (C) Number of escape responses over the 15 min observation segment. (D) Number of attack of inanimate objects over the 15 min observation segment. Gray bars show mean + standard error for baseline sessions, red bars show mean + standard error for bicuculline microinjection sessions. Symbols represent individual cases. ${ }^{*} P<0.05$ (baseline vs. bicuculline infused). JA received $2.5 \mathrm{nmol}$, ZK received $2.5 \mathrm{nmol}$, GW-30 received $5 \mathrm{nmol}$, and GW- 4 received $7 \mathrm{nmol}$ of bicuculline methiodide into the PAG.

during the baseline session. Thus, a positive value indicates increased asymmetry in a particular direction, whereas a negative value indicates a reduced asymmetry. A value of zero indicates no change in asymmetry between baseline and infused sessions. These were calculated separately for ipsiversive and contraversive behaviors (relative to the site of PAG injection). Data were analyzed for 5 of the 6 cases (motor behavior for GW-30 was unavailable for analysis). We found that the duration of ipsiversive asymmetry was significantly decreased after infusion of PAG with bicuculline and that the duration of contraversive asymmetry was significantly increased, both with respect to baseline (i.e., a difference score of zero). These effects were revealed by one sample $t$-tests $(P s=0.03$ and 0.002 , respectively). 


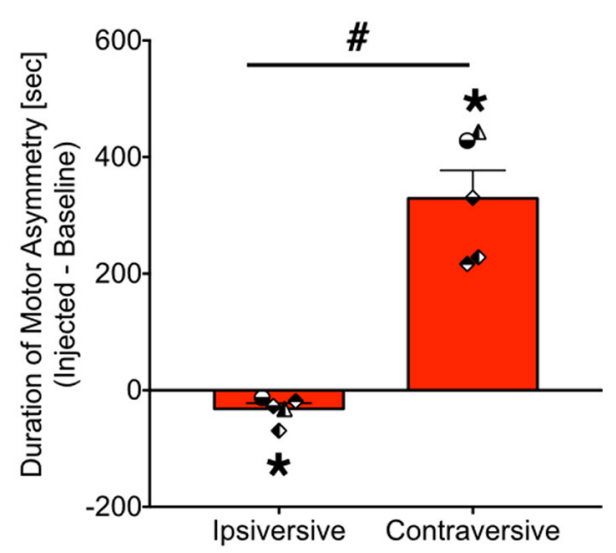

FIGURE 4 | Bicuculline microinjection in PAG evokes contraversive motor/postural asymmetry. Bars show the net duration (seconds) of motor asymmetry after bicuculline microinfusion minus the duration of motor asymmetry under baseline conditions [mean + standard error]. Ipsiversive asymmetry indicates motor and posture toward the same side as the injection, whereas contraversive indicates motor and posture directed away from the side of injection. ${ }^{*} P<0.05$ as compared to zero (no asymmetry). $\# P<0.05$ contraversive as compared to ipsiversive. Symbols show individual animals, and correspond to the legend in Figure 3. JA received $2.5 \mathrm{nmol}$, ZK received $2.5 \mathrm{nmol}$, GW-30 received $5 \mathrm{nmol}$, and GW-4 received $7 \mathrm{nmol}$ of bicuculline methiodide into the PAG.

\section{DISCUSSION}

Here we report the occurrence of defense-associated vocalizations after activation of the PAG with the GABA-A receptor antagonist, bicuculline methiodide. The volume of tissue activated by our microinjections likely spanned a portion of both the lateral and dorsolateral PAG. Given the relatively short amount of time between our microinjections and the onset of behavioral responses (i.e., $0-15 \mathrm{~min}$ ), it is likely that 0.5 to 1 $\mathrm{mm}$ of tissue was activated. This volume of spread is consistent with prior reports employing gadolinium microinjection from our laboratory (Des)ardin et al., 2013; Forcelli et al., 2014) and with the spread of isotopically labeled bicuculline reported by others (Yoshida et al., 1991). The vocalizations evoked from PAG are consistent with prior reports employing electrical stimulation within the PAG (e.g., Larson and Kistler, 1986; Larson, 1991; Larson et al., 1994) of macaques and chemical stimulation of the PAG in the squirrel monkey (Jürgens and Lu, 1993; Lu and Jürgens, 1993). While activation of either the PAG (present study) or DLSC (prior report; DesJardin et al., 2013) induce clear motor/postural asymmetry and defense-associated vocalizations, there was a dissociation between these structures with respect to motor defensive behaviors. While activation of the DLSC induced cowering, escape and attack of inanimate objects, none of these behaviors were observed after activation of PAG.

Microinjection of bicuculline into the DLSC in our prior studies produced cowering, vocalizations, escape responses and attack of inanimate objects. These behaviors were never observed with low-dose bicuculline (e.g., $2.5 \mathrm{nmol}$ ), but rather emerged when higher doses were delivered. Indeed, the lowest effective dose for evoking defense responses from the DLSC was $4.6 \mathrm{nmol}$
(DesJardin et al., 2013). Here, with doses of bicuculline as low as $2.5 \mathrm{nmol}$, vocalizations emerged, and were evoked in all cases. By contrast, after DLSC activation vocalizations were present in only 7 of 9 cases (DesJardin et al., 2013). In the absence of a full dose response for bicuculline microinjection into the PAG, we cannot rule out the possibility that higher doses would have evoked escape behaviors. However, when vocalizations were evoked from DLSC, they always co-occurred with other defense responses, whereas in the PAG, vocalizations occurred without gross motor defense reactions.

\section{Vocalizations}

It has previously been reported in macaques that electrical stimulation in a site within the lateral PAG, comparable to ours, produced inhibition within the spinothalamic tract, perhaps related to analgesia (Gerhart et al., 1984). A similar profile has been reported after GABA antagonist or glycine antagonist injection into lateral PAG of monkeys (Lin et al., 1994). However, because animals were intubated and anesthetized during these experiments, it is perhaps unsurprising that no comment was made regarding the presence or quality of vocalizations or emergence of defensive responses. In the cat, PAG neurons project to the nucleus ambiguus and nucleus retroambiguus (Holstege, 1989); a similar topography is likely in the macaque, in which second order neurons project from nucleus retroambiguus to laryngeal motor neurons (VanderHorst et al., 2001). These connections position the PAG as a likely candidate to mediate defense-related vocalizations. Neurophysiological recordings in macaques have demonstrated that neurons in the lateral PAG burst fire shortly before or during the onset of spontaneous vocalizations and cease firing during or just after a vocalization ends (Larson and Kistler, 1986). Similarly, respiratory-related neurons within the PAG burst during the inspiratory phase of respiration. Of interest, however, these cells have been described to fire during both spontaneous "coo" vocalizations as well as "bark" vocalizations (Larson, 1991). Here, coo vocalizations were never evoked after activation of PAG, indeed in the three cases in which affiliative vocalizations (such as coos) were observed under baseline conditions, they were completely abolished after activation of PAG.

The PAG may represent part of a final common pathway mediating defensive vocalizations. For example, defensiveassociated hissing responses can be evoked by stimulation of the PAG in cats (Carrive et al., 1987; Bandler and Carrive, 1988; Wang et al., 2002). While similar responses can be evoked from the medial hypothalamus, these responses require the PAG: pre-treatment of the PAG with NMDA receptor antagonists abolishes the hypothalamic-evoked hissing responses (Schubert et al., 1996). Similarly, the PAG may play a role in defensive vocalizations evoked from other structures (e.g., the DLSC). It is thus possible that the defense-associated vocalizations we observed after activation of the DLSC, may likewise require the PAG. In support of the idea that these two structures are functionally interconnected, neurons within the PAG are modulated during spontaneous eye saccades in macaques, a behavior closely associated with and requiring the DLSC (Kase et al., 1986). Moreover, the deep layers of the SC project to the 
lateral PAG in the rat (Beitz, 1982). Whether inhibition of PAG will attenuate SC-evoked vocalizations remains to be tested.

While the nature of vocalizations evoked from the PAG has not previously been characterized in macaques, in squirrel monkeys even lower amounts of bicuculline than those used in the present study have been tested $(0.1-1 \mathrm{nmol}$, threshold dose) (Lu and Jürgens, 1993). Injections of bicuculline into the PAG of squirrel monkeys evoked vocalizations with latencies similar to those that we report here (seconds to tens of minutes), with responses lasting for minutes to hours ( $\mathrm{Lu}$ and Jürgens, 1993). Sites throughout the dorsoventral extent of the lateral PAG produced vocalizations. Common vocalizations included "peeps" which are alert calls, squeaks, which are frustration calls, and shrieks, which indicate defensive threat (Lu and Jürgens, 1993). Trill calls, which are primarily positive and emitted in response to pleasurable events in squirrel monkeys rarely occurred after PAG activation. Indeed, trills were seen only in 2 of 28 cases following GABA antagonist injection (Lu and Jürgens, 1993). Interestingly, in a subset of sites, the investigators injected in the DLSC, rather than the PAG. As is our experience in macaques, injections into the DLSC also evoked vocalizations.

In the cat, electrical stimulation, or injection of exictatory amino acids into the PAG has also been associated with defenserelated vocalizations. For example, stimulation of the lateral PAG (akin to the site we stimulated in the monkey) triggered hissing and ear retraction. Stimulation of the ventrolateral PAG (deeper than we injected) also evoked howling and growling, piloerection, and back arching. Interestingly, visual and tactile stimulation following kainate microinjection into the PAG elicited attack behaviors in 3 of 4 cats (Bandler and Carrive, 1988). Within the PAG of the cat, some evidence for topograpic organization of vocalization-evoking regions exists. For example, activation of the rostral PAG preferentially evoked hissing and growling, while activation of the posterior PAG preferentially evoked howling behavior. Moreover, while lateral and dorsal sites along the rostrocaudal axis evoked defensive vocalizations including hissing and growling, medial sites evoked meowing, crying and screaming vocalizations (Wang et al., 2002). In almost all cases, these pharmacological activations triggered an increase in mean arterial pressure (Wang et al., 2002). These behaviors do not require the telencephalon, as they are evident in the decerebrate preparation (Carrive et al., 1987). This hypertensive response is considered a key part of defense reactions evoked from the PAG in cats; it has been suggested that this is mediated by direct projections to a region of the ventrolateral medulla, the subretrofacial nucleus (Carrive et al., 1988). In macaques, the ventrolateral medulla likewise receives input from the PAG, and in particular the lateral PAG (in the approximate zone that we injected in the present study) (VanderHorst et al., 2001). Thus, the degree to which our manipulations would likewise result in hypertensive responses, while unstudied, seems plausible.

\section{Motor Responses}

In the present study, activation of the lateral PAG produced a strong motor/postural asymmetry: bicuculline increased asymmetry by an average of 11 -fold across subjects. Neurons within the lateral PAG project to the so called medial pontomedullary head-movement region, perhaps providing an anatomical substrate for orienting responses to threatening stimuli (Cowie et al., 1994). Our data in macaques are consistent with reports in other species; for example in rats, lateralized defensive reactions have been reported after PAG activation, including a "defensive sideways" posture, characterized by concave body position contralateral to the injection (Depaulis et al., 1989); this is similar to the defensive posture seen following activation of the DLSC (Sahibzada et al., 1986). Similarly, in cats, injections of excitatory amino acids into the lateral PAG evokes escape movements (rearing, pawing) and jumping responses and lateralized posture (circling, head turning). It is further worth noting that sites that evoked vocalization in the cat were preferentially associated with head turning behavior and escapes (Carrive et al., 1988; Zhang et al., 1990). Despite evoking postural asymmetry, we failed to reliably evoke other motor defense responses in the macaque.

Interestingly, in addition to motor defense reactions, vocalizations, and postural asymmetry, immobility responses have been reported in several species after PAG activation. For example, optogenetic activation of the lateral PAG in the rat induces both freezing, and flight behaviors, with higher irradiance needed to evoke flight as compared to freezing behavior (Assareh et al., 2016). Interestingly, these thresholds were lower in the lateral PAG as compared to the ventrolateral PAG. In the guinea pig, tonic immobility responses are differentially modulated by the dorsal and ventral PAG, with activation of the former reducing tonic immobility and activation of the latter increasing it (LeitePanissi et al., 2003; Ramos Coutinho et al., 2008). A similar pattern has been reported in the cat, where ventral PAG activation triggers increased immobility (Zhang et al., 1990). Here we did not observe increased immobility after lateral PAG activation, however, the degree to which this response might be evoked by activation of other sites within the PAG (e.g., the ventral or ventrolateral PAG) remains to be determined. It is also possible that freezing responses may be of preferential benefit for prey animals (e.g., rodents) as compared to primates.

\section{CONCLUSIONS}

Here we have reported that activation of the lateral/dorsolateral PAG evoked striking defense-related vocalizations and postural asymmetry in macaques. Based on data in the rat, cat, squirrel monkey and guinea pig, we hypothesized that we would evoke both defensive responses and vocalizations (Bandler and Carrive, 1988; Carrive et al., 1988; Depaulis et al., 1989; Jürgens and Lu, 1993; Lu and Jürgens, 1993; Ramos Coutinho et al., 2008; Assareh et al., 2016). To our surprise, defense responses were not common after activation of the lateral/dorsolateral PAG in the macaque. These data provide a dissociation between the pattern of defensive responses evoked from the DLSC and the PAG, suggesting an anatomical specialization within the midbrain circuitry controlling fear. While activation of 
either structure triggered vocalizations and asymmetry, the PAG showed a lower threshold for evoking vocalizations, and only the DLSC triggered appreciable motor defense reactions.

The PAG is structurally and functionally conserved across phylogeny. In primate species, where there is an elaboration of neocortical areas, the contribution of top-down influence over PAG function in defense responses may be of importance. Indeed, the PAG receives input from the neocortex, including dosolateral and orbital frontal regions, and particularly dense input from medial prefrontal regions, including the cingulate cortex (Bandler and McCulloch, 1984; Bandler et al., 1985b; Falconi-Sobrinho et al., 2017). Similarly, premotor cortex sends extensive projections to the PAG, and in particular the lateral PAG (An et al., 1998). While direct activation of the PAG (e.g., by bicuculline microinfusion) would bypass any top-down control, in the normally behaving animal, a role for high-level cortical input must be considered.

\section{REFERENCES}

An, X., Bandler, R., Ongür, D., and Price, J. L. (1998). Prefrontal cortical projections to longitudinal columns in the midbrain periaqueductal gray in macaque monkeys. J. Comp. Neurol. 401, 455-479. doi: 10.1002/(SICI) 1096-9861(19981130)401:4<455::AID-CNE3>3.0.CO;2-6

Assareh, N., Sarrami, M., Carrive, P., and McNally, G. P. (2016). The organization of defensive behavior elicited by optogenetic excitation of rat lateral or ventrolateral periaqueductal gray. Behav. Neurosci. 130, 406-414. doi: $10.1037 /$ bne 0000151

Bandler, R., and Carrive, P. (1988). Integrated defence reaction elicited by excitatory amino acid microinjection in the midbrain periaqueductal grey region of the unrestrained cat. Brain Res. 439, 95-106. doi: 10.1016/0006-8993(88)91465-5

Bandler, R., Depaulis, A., and Vergnes, M. (1985a). Identification of midbrain neurones mediating defensive behaviour in the rat by microinjections of excitatory amino acids. Behav. Brain Res. 15, 107-119. doi: 10.1016/0166-4328(85)90058-0

Bandler, R., and McCulloch, T. (1984). Afferents to a midbrain periaqueductal grey region involved in the "defense reaction" in the cat as revealed by horseradish peroxidase. II. The diencephalon. Behav. Brain Res. 13, 279-285. doi: 10.1016/0166-4328(84)90171-2

Bandler, R., McCulloch, T., and Dreher, B. (1985b). Afferents to a midbrain periaqueductal grey region involved in the "defence reaction" in the cat as revealed by horseradish peroxidase. I. The telencephalon. Brain Res. 330, 109-119. doi: 10.1016/0006-8993(85)90011-3

Beitz, A. J. (1982). The organization of afferent projections to the midbrain periaqueductal gray of the rat. Neuroscience 7, 133-159. doi: 10.1016/0306-4522(82)90157-9

Brandão, M. L., Cardoso, S. H., Melo, L. L., Motta, V., and Coimbra, N. C. (1994). Neural substrate of defensive behavior in the midbrain tectum. Neurosci. Biobehav. Rev. 18, 339-346. doi: 10.1016/0149-7634(94) 90047-7

Brandão, M. L., Troncoso, A. C., de Souza Silva, M. A., and Huston, J. P. (2003). The relevance of neuronal substrates of defense in the midbrain tectum to anxiety and stress: empirical and conceptual considerations. Eur. J. Pharmacol. 463, 225-233. doi: 10.1016/S0014-2999(03)01284-6

Carrive, P., Bandler, R., and Dampney, R. A. (1988). Anatomical evidence that hypertension associated with the defence reaction in the cat is mediated by a direct projection from a restricted portion of the midbrain periaqueductal grey to the subretrofacial nucleus of the medulla. Brain Res. 460, 339-345. doi: 10.1016/0006-8993(88)90378-2

\section{AUTHOR CONTRIBUTIONS}

PF designed the study, conducted experiments, analyzed data, and wrote the manuscript. HW analyzed data and wrote the manuscript. LM designed the study, conducted experiments, analyzed data, and wrote the manuscript.

\section{FUNDING}

The study was supported in part by R01 MH084069 (LM), R01 MH082364 (LM), K02HD042269 (LM), Cure Autism Now (now Autism speaks; LM), National Alliance for Autism Research (NAAR; now Autism speaks; LM), and KL2TR001432 (PF).

\section{ACKNOWLEDGMENTS}

The authors wish to thank Carrie Silver, Jackie DesJardin, and Laurie Wellman for technical assistance.

Carrive, P., Dampney, R. A., and Bandler, R. (1987). Excitation of neurones in a restricted portion of the midbrain periaqueductal grey elicits both behavioural and cardiovascular components of the defence reaction in the unanaesthetised decerebrate cat. Neurosci. Lett. 81, 273-278. doi: 10.1016/0304-3940(87)90395-8

Cattarelli, M., and Chanel, J. (1979). Influence of some biologically meaningful odorants on the vigilance states of the rat. Physiol. Behav. 23, 831-838. doi: 10.1016/0031-9384(79)90186-0

Coimbra, N. C., De Oliveira, R., Freitas, R. L., Ribeiro, S. J., Borelli, K. G., Pacagnella, R. C., et al. (2006). Neuroanatomical approaches of the tectum-reticular pathways and immunohistochemical evidence for serotonin-positive perikarya on neuronal substrates of the superior colliculus and periaqueductal gray matter involved in the elaboration of the defensive behavior and fear-induced analgesia. Exp. Neurol. 197, 93-112. doi: 10.1016/j.expneurol.2005.08.022

Coker-Appiah, D. S., White, S. F., Clanton, R., Yang, J., Martin, A., and Blair, R. J. R. (2013). Looming animate and inanimate threats: the response of the amygdala and periaqueductal gray. Soc. Neurosci. 8, 621-630. doi: 10.1080/17470919.2013.839480

Cowie, R. J., Smith, M. K., and Robinson, D. L. (1994). Subcortical contributions to head movements in macaques. II. Connections of a medial pontomedullary head-movement region. J. Neurophysiol. 72, 2665-2682.

Dean, P., Redgrave, P., Sahibzada, N., and Tsuji, K. (1986). Head and body movements produced by electrical stimulation of superior colliculus in rats: effects of interruption of crossed tectoreticulospinal pathway. Neuroscience 19, 367-380. doi: 10.1016/0306-4522(86)90267-8

Dean, P., Redgrave, P., and Westby, G. W. (1989). Event or emergency? Two response systems in the mammalian superior colliculus. Trends Neurosci. 12, 137-147. doi: 10.1016/0166-2236(89)90052-0

De Franceschi, G., Vivattanasarn, T., Saleem, A. B., and Solomon, S. G. (2016). Vision guides selection of freeze or flight defense strategies in mice. Curr. Biol. 26, 2150-2154. doi: 10.1016/j.cub.2016.06.006

Depaulis, A., Bandler, R., and Vergnes, M. (1989). Characterization of pretentorial periaqueductal gray matter neurons mediating intraspecific defensive behaviors in the rat by microinjections of kainic acid. Brain Res. 486, 121-132. doi: 10.1016/0006-8993(89)91284-5

DesJardin, J. T., Holmes, A. L., Forcelli, P. A., Cole, C. E., Gale, J. T., Wellman, L. L., et al. (2013). Defense-like behaviors evoked by pharmacological disinhibition of the superior colliculus in the primate. J. Neurosci. 33, 150-155. doi: 10.1523/JNEUROSCI.2924-12.2013

Dybdal, D., Forcelli, P. A., Dubach, M., Oppedisano, M., Holmes, A., Malkova, L., et al. (2013). Topography of dyskinesias and torticollis evoked by 
inhibition of substantia nigra pars reticulata. Mov. Disord. 28, 460-468. doi: $10.1002 / \mathrm{mds} .25215$

Eichenberger, G. C. D., Ribeiro, S. J., Osaki, M. Y., Maruoka, R. Y., Resende, G. C. C., Castellan-Baldan, L., et al. (2002). Neuroanatomical and psychopharmacological evidence for interaction between opioid and GABAergic neural pathways in the modulation of fear and defense elicited by electrical and chemical stimulation of the deep layers of the superior colliculus and dorsal periaqueductal gray matter. Neuropharmacology 42, 48-59. doi: 10.1016/S0028-3908(01)00155-1

Falconi-Sobrinho, L. L., dos Anjos-Garcia, T., Elias-Filho, D. H., and Coimbra, N. C. (2017). Unravelling cortico-hypothalamic pathways regulating unconditioned fear-induced antinociception and defensive behaviours. Neuropharmacology 113(Pt A), 367-385. doi: 10.1016/j.neuropharm.2016.10.001

Forcelli, P. A., DesJardin, J. T., West, E. A., Holmes, A. L., Elorette, C., Wellman, L. L., et al. (2016). Amygdala selectively modulates defensive responses evoked from the superior colliculus in non-human primates. Soc. Cogn. Affect. Neurosci. 11, 2009-2019. doi: 10.1093/scan/nsw111

Forcelli, P. A., Palchik, G., Leath, T., Desjardin, J. T., Gale, K., and Malkova, L. (2014). Memory loss in a nonnavigational spatial task after hippocampal inactivation in monkeys. Proc. Natl. Acad. Sci. U.S.A. 111, 4315-4320. doi: 10.1073/pnas.1320562111

Forcelli, P. A., Wellman, L. L., and Malkova, L. (2017). Blockade of glutamatergic transmission in the primate basolateral amygdala suppresses active behavior without altering social interaction. Behav. Neurosci. 131, 192-200. doi: 10.1037/bne0000187

Fox, A. S., Shelton, S. E., Oakes, T. R., Davidson, R. J., and Kalin, N. H. (2008). Trait-like brain activity during adolescence predicts anxious temperament in primates. PLoS ONE 3:e2570. doi: 10.1371/journal.pone.0002570

Gerhart, K. D., Yezierski, R. P., Wilcox, T. K., and Willis, W. D. (1984). Inhibition of primate spinothalamic tract neurons by stimulation in periaqueductal gray or adjacent midbrain reticular formation. J. Neurophysiol. 51, 450-466.

Holmes, A. L., Forcelli, P. A., DesJardin, J. T., Decker, A. L., Teferra, M., West, E. A., et al. (2012). Superior colliculus mediates cervical dystonia evoked by inhibition of the substantia nigra pars reticulata. J. Neurosci. 32, 13326-13332. doi: 10.1523/JNEUROSCI.2295-12.2012

Holstege, G. (1989). Anatomical study of the final common pathway for vocalization in the cat. J. Comp. Neurol. 284, 242-252. doi: 10.1002/cne.902840208

Izquierdo, A., and Murray, E. A. (2004). Combined unilateral lesions of the amygdala and orbital prefrontal cortex impair affective processing in rhesus monkeys. J. Neurophysiol. 91, 2023-2039. doi: 10.1152/jn.00968.2003

Jürgens, U., and Lu, C. L. (1993). Interactions between glutamate, GABA, acetylcholine and histamine in the periaqueductal gray's control of vocalization in the squirrel monkey. Neurosci. Lett. 152, 5-8. doi: 10.1016/0304-3940(93)90469-2

Kase, M., Nagata, R., and Kato, M. (1986). Saccade-related activity of periaqueductal gray matter of the monkey. Invest. Ophthalmol. Vis. Sci. 27, 1165-1169.

Kawai, N., and Koda, H. (2016). Japanese monkeys (Macaca fuscata) quickly detect snakes but not spiders: evolutionary origins of fear-relevant animals. J. Comp. Psychol. 130, 299-303. doi: 10.1037/com0000032

Krieger, J. E., and Graeff, F. G. (1985). Defensive behavior and hypertension induced by glutamate in the midbrain central gray of the rat. Braz. J. Med. Biol. Res. 18, 61-67.

Larson, C. R. (1991). On the relation of PAG neurons to laryngeal and respiratory muscles during vocalization in the monkey. Brain Res. 552, 77-86. doi: 10.1016/0006-8993(91)90662-F

Larson, C. R., and Kistler, M. K. (1986). The relationship of periaqueductal gray neurons to vocalization and laryngeal EMG in the behaving monkey. Exp. Brain Res. 63, 596-606. doi: 10.1007/BF00237482

Larson, C. R., Yajima, Y., and Ko, P. (1994). Modification in activity of medullary respiratory-related neurons for vocalization and swallowing. J. Neurophysiol. 71, 2294-2304.

Leite-Panissi, C. R., Coimbra, N. C., and Menescal-de-Oliveira, L. (2003). The cholinergic stimulation of the central amygdala modifying the tonic immobility response and antinociception in guinea pigs depends on the ventrolateral periaqueductal gray. Brain Res. Bull. 60, 167-178. doi: 10.1016/S0361-9230(03)00031-5

Lin, Q., Peng, Y., and Willis, W. D. (1994). Glycine and GABAA antagonists reduce the inhibition of primate spinothalamic tract neurons produced by stimulation in periaqueductal gray. Brain Res. 654, 286-302. doi: 10.1016/0006-8993(94)90491-X

Lu, C. L., and Jürgens, U. (1993). Effects of chemical stimulation in the periaqueductal gray on vocalization in the squirrel monkey. Brain Res. Bull. 32, 143-151. doi: 10.1016/0361-9230(93)90068-M

Malkova, L., Forcelli, P. A., Wellman, L. L., Dybdal, D., Dubach, M. F., and Gale, K. (2015). Blockade of glutamatergic transmission in perirhinal cortex impairs object recognition memory in macaques. J. Neurosci. 35, 5043-5050. doi: 10.1523/JNEUROSCI.4307-14.2015

Mobbs, D., Yu, R., Rowe, J. B., Eich, H., FeldmanHall, O., and Dalgleish, T. (2010). Neural activity associated with monitoring the oscillating threat value of a tarantula. Proc. Natl. Acad. Sci. U.S.A. 107, 20582-20586. doi: 10.1073/pnas.1009076107

Nashold, B. S., Wilson, W. P., and Slaughter, D. G. (1969). Sensation evoked by stimulation in the midbrain of main. J. Neurosurg. 30, 14-24. doi: 10.3171/jns.1969.30.1.0014

National Research Council U.S., Institute for Laboratory Animal Research U.S., and National Academies Press U.S. (2011). Guide for the Care and Use of Laboratory Animals, 8th Edn. Washington, DC: National Academies Press.

Novak, M. F., and Sackett, G. P. (1997). Pair-rearing infant monkeys (Macaca nemestrina) using a "rotating-peer" strategy. Am. J. Primatol. 41, 141-149. doi: 10.1002/(SICI)1098-2345(1997)41:2<141::AID-AJP6>3.0.CO;2-X

Ramos Coutinho, M., da Silva, L. F. S., and Menescal-de-Oliveira, L. (2008). Modulation of tonic immobility in guinea pig PAG by homocysteic acid, a glutamate agonist. Physiol. Behav. 94, 468-473. doi: 10.1016/j.physbeh.2008.02.013

Sahibzada, N., Dean, P., and Redgrave, P. (1986). Movements resembling orientation or avoidance elicited by electrical stimulation of the superior colliculus in rats. J. Neurosci. 6, 723-733.

Satpute, A. B., Wager, T. D., Cohen-Adad, J., Bianciardi, M., Choi, J.-K., Buhle, J. T., et al. (2013). Identification of discrete functional subregions of the human periaqueductal gray. Proc. Natl. Acad. Sci. U.S.A. 110, 17101-17106. doi: 10.1073/pnas.1306095110

Schenberg, L. C., Póvoa, R. M. F., Costa, A. L. P., Caldellas, A. V., Tufik, S., and Bittencourt, A. S. (2005). Functional specializations within the tectum defense systems of the rat. Neurosci. Biobehav. Rev. 29, 1279-1298. doi: 10.1016/j.neubiorev.2005.05.006

Schubert, K., Shaikh, M. B., and Siegel, A. (1996). NMDA receptors in the midbrain periaqueductal gray mediate hypothalamically evoked hissing behavior in the cat. Brain Res. 726, 80-90. doi: 10.1016/0006-8993(96)00261-2

Shang, C., Liu, Z., Chen, Z., Shi, Y., Wang, Q., Liu, S., et al. (2015). BRAIN CIRCUITS. A parvalbumin-positive excitatory visual pathway to trigger fear responses in mice. Science 348, 1472-1477. doi: 10.1126/science. aaa8694

Shibasaki, M., Nagumo, S., and Koda, H. (2014). Japanese monkeys (Macaca fuscata) spontaneously associate alarm calls with snakes appearing in the left visual field. J. Comp. Psychol. 128, 332-335. doi: 10.1037/a0036049

Ullah, F., dos Anjos-Garcia, T., dos Santos, I. R., Biagioni, A. F., and Coimbra, N. C. (2015). Relevance of dorsomedial hypothalamus, dorsomedial division of the ventromedial hypothalamus and the dorsal periaqueductal gray matter in the organization of freezing or oriented and non-oriented escape emotional behaviors. Behav. Brain Res. 293, 143-152. doi: 10.1016/j.bbr.2015.07.013

VanderHorst, V. G., Terasawa, E., and Ralston, H. J. (2001). Monosynaptic projections from the nucleus retroambiguus region to laryngeal motoneurons in the rhesus monkey. Neuroscience 107, 117-125. doi: 10.1016/S0306-4522(01)00343-8

Wallace, K. J., and Rosen, J. B. (2000). Predator odor as an unconditioned fear stimulus in rats: elicitation of freezing by trimethylthiazoline, a component of fox feces. Behav. Neurosci. 114, 912-922. doi: 10.1037/0735-7044.114.5.912

Wang, M. R., Kuo, J. S., and Chai, C. Y. (2002). Cardiovascular and vocalization reactions elicited by $\mathrm{N}$-methyl-D-aspartate in the pretentorial periaqueductal grey of cats. Clin. Exp. Pharmacol. Physiol. 29, 759-771. doi: 10.1046/j.1440-1681.2002.03728.x 
Weiss, L., Brandl, P., and Frynta, D. (2015). Fear reactions to snakes in naïve mouse lemurs and pig-tailed macaques. Primates 56, 279-284. doi: 10.1007/s10329-015-0473-3

Wellman, L. L., Forcelli, P. A., Aguilar, B. L., and Malkova, L. (2016). Bidirectional control of social behavior by activity within basolateral and central amygdala of primates. J. Neurosci. 36, 8746-8756. doi: 10.1523/JNEUROSCI.033316.2016

Wellman, L. L., Gale, K., and Malkova, L. (2005). GABAA-mediated inhibition of basolateral amygdala blocks reward devaluation in macaques. J. Neurosci. 25, 4577-4586. doi: 10.1523/JNEUROSCI.2257-04.2005

West, E. A., DesJardin, J. T., Gale, K., and Malkova, L. (2011). Transient inactivation of orbitofrontal cortex blocks reinforcer devaluation in macaques. J. Neurosci. 31, 15128-15135. doi: 10.1523/JNEUROSCI.3295-11.2011

Yilmaz, M., and Meister, M. (2013). Rapid innate defensive responses of mice to looming visual stimuli. Curr. Biol. 23, 2011-2015. doi: 10.1016/j.cub.2013.08.015

Yoshida, M., Nagatsuka, Y., Muramatsu, S., and Niijima, K. (1991). Differential roles of the caudate nucleus and putamen in motor behavior of the cat as investigated by local injection of GABA antagonists. Neurosci. Res. 10, 34-51. doi: 10.1016/0168-0102(91)90018-T

Zhang, S. P., Bandler, R., and Carrive, P. (1990). Flight and immobility evoked by excitatory amino acid microinjection within distinct parts of the subtentorial midbrain periaqueductal gray of the cat. Brain Res. 520, 73-82. doi: 10.1016/0006-8993(90)91692-A

Conflict of Interest Statement: The authors declare that the research was conducted in the absence of any commercial or financial relationships that could be construed as a potential conflict of interest.

Copyright (c) 2017 Forcelli, Waguespack and Malkova. This is an open-access article distributed under the terms of the Creative Commons Attribution License (CC BY). The use, distribution or reproduction in other forums is permitted, provided the original author(s) or licensor are credited and that the original publication in this journal is cited, in accordance with accepted academic practice. No use, distribution or reproduction is permitted which does not comply with these terms. 